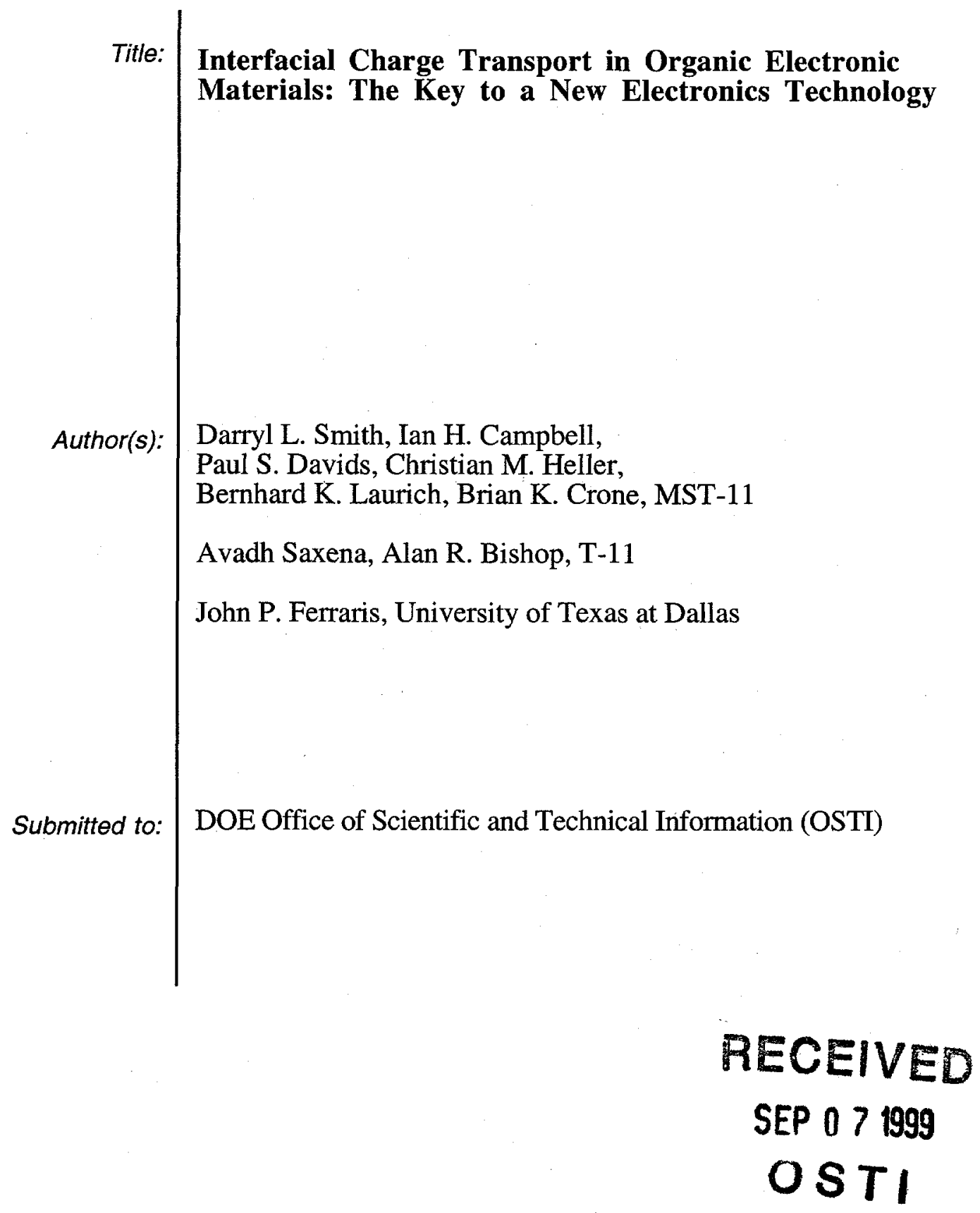

Los Alamos National Laboratory, an affirmative action/equal opportunity employer, is operated by the University of California for the U.S. Department of Energy under contract W-7405-ENG-36. By acceptance of this article, the publisher recognizes that the U.S. Government retains a nonexclusive, royaltyfree license to publish or reproduce the published form of this contribution, or to allow others to do so, for U.S. Government purposes. Las Alamos National Laboratory requests that the publisher identify this article as work performed under the auspices of the U.S. Department of Energy. Los Alamos National Laboratory strongly supports academic freedom and a researcher's right to publish; as an institution, however, the Laboratory does not endorse the viewpoint of a publication or guarantee its technical correctness. 


\section{DISCLAIMER}

This report was prepared as an account of work sponsored by an agency of the United States Government. Neither the United States Government nor any agency thereof, nor any of their employees, make any warranty, express or implied, or assumes any legal liability or responsibility for the accuracy, completeness, or usefulness of any information, apparatus, product, or process disclosed, or represents that its use would not infringe privately owned rights. Reference herein to any specific commercial product, process, or service by trade name, trademark, manufacturer, or otherwise does not necessarily constitute or imply its endorsement, recommendation, or favoring by the United States Government or any agency thereof. The views and opinions of authors expressed herein do not necessarily state or reflect those of the United States Government or any agency thereof. 


\section{DISCLAIMER}

Portions of this document may be illegible in electronic image products. Images are produced from the best available original document. 


\title{
Interfacial Charge Transport in Organic Electronic Materials: The Key to a New Electronics Technology
}

\author{
*Darryl L. Smith, Ian H. Campbell, Paul S. Davids, \\ Christian M. Heller, Bernhard K. Laurich and Brian K. Crone, MST-11 \\ Avadh Saxena, Alan R. Bishop and Zhi G. Yu, T-11 \\ John P. Ferraris, University of Texas at Dallas
}

\begin{abstract}
This is the final report of a three-year, Laboratory Directed Research and Development (LDRD) project at Los Alamos National Laboratory (LANL). The primary aim of this project is to obtain a basic scientific understanding of electrical transport processes at interfaces that contain an organic electronic material. Because of their processing advantages and the tunability of their electronic properties, organic electronic materials are revolutionizing major technological areas such as information display. We completed an investigation of the fundamental electronic excitation energies in the prototype conjugated polymer MEH-PPV. We completed a combined theoretical/experimental study of the energy relation between charged excitations in a conjugated polymer and the metal at a polymer/metal interface. We developed a theoretical model that explains injection currents at polymer/metal interfaces. We have made electrical measurements on devices fabricated using the conjugated polymer MEH-PPV and a series of metals.
\end{abstract}

\section{Background and Research Objectives}

The primary aim of the project is to obtain a basic scientific understanding of electrical transport processes at interfaces that contain an organic electronic material as a constituent. These interfaces are the essential active elements of every organic electronic and electro-optic device. Because of their processing advantages, the tunability of their electronic properties and their flexibility in both materials and device design, organic electronic materials and devices are revolutionizing major technological areas such as information display and optical communication. There are a great many design options available for organic electronic materials and devices both at the molecular and mesoscopic scales. When this project started, the basic understanding of the properties of these materials and devices was insufficient to allow the design options to be intelligently utilized. Progress was limited by a lack of

*Principal Investigator, e-mail: dsmith@lanl.gov 
fundamental understanding. Because interfaces are the essential active elements in every organic electronic device, understanding interfacial charge transport was the key to realizing the potential of these materials and devices.

Two basic classes of questions can be asked about interfaces involving organic electronic materials: 1) the relative positions of energy levels in the two constituent materials forming the interface, and 2) cross sections for charged excitation transfer across the interface. We investigated metal/organic interfaces and organic/organic heterojunctions. These are the two essential kinds of interfaces in organic electronic and electro-optic devices. We studied these phenomena using a combination of experimental and theoretical methods.

Our specific goals were:

1. To determine relative energy levels and cross sections for charged excitation injection at metal/organic interfaces using Ballistic Electron Emission Microscopy (BEEM), to theoretically describe the BEEM technique as applied to organic materials, and to determine how relative energy levels and cross sections for the various possible injection processes depend on the properties of the organic material.

2. To investigate tunneling through thin organic layers in metal/thin-organic/metal structures, experimentally and theoretically, and to determine the relative importance of various tunneling channels for different organic electronic materials.

3. To determine the relative energy levels of charged excitations at organic heterojunctions using hot electron luminescence techniques combined with theoretical analysis and to determine how these energy levels depend on the properties of the constituent organic materials.

Organic electronic materials and devices are revolutionizing key technological areas, including information display and optical communications. Major advantages of organic materials are their ability to be processed economically in large area, the tunability of their electronic properties, and their flexibility in both materials and device design. These materials can be designed and built at the molecular level, using the methods of organic synthesis, and devices can be designed and built at the mesoscopic level using device fabrication techniques. There are a great many design options available, both at the microscopic and mesoscopic level. We did not understand the properties of these materials or the operation of the devices fabricated from them well enough to systematically utilize the wide range of design options available. Progress was limited by a lack of understanding of fundamental physical principles. The work described here provides the basic scientific understanding of the most critical issue for organic electronic devices-electronic transport processes at organic interfaces.

Light emitting diodes (LEDs) are the most successful organic-based electronic devices. Recent improvements in the performance of these devices have led to the commercial licensing 
of polymer LED technology and to the initial production of these devices. The discovery of polymer LEDs was largely serendipitous. There has been considerable improvement in these devices since their original discovery and their performance has now reached the point where devices are beginning to be commercially produced. Charge injection at metal/organic interfaces is one of the most important processes occurring in any organic electronic device and it is essential to obtain a realistic description of this process in order to design organic electronic materials and devices.

The electronic properties of organic electronic materials are completely different than those of the single crystal semiconductors that are conventionally used to make electronic devices. Organic electronic materials are flexible (strong electron-phonon interaction), polarizible, correlated (strong electron-electron interaction) disordered systems, whereas conventional semiconductors have rigid crystal structures and weak electron-phonon and electron-electron interactions. As a result of this difference in material properties, the design principles for organic electronic devices are completely different than those for conventional semiconductor devices. We are beginning to understand these device design principles, and a number of points have become clear. For example, because of the relatively low mobility of organic materials, devices must be designed using vertical structures where the conduction is through thin layers. Analogs of field effect transistors, which are lateral devices that require relatively high mobility, have not been successful when fabricated from organic materials. Vertical device designs, which do not require high mobility, are much more promising. Interfaces are the essential active structure in vertical devices designed from organic electronic materials. Two kinds of interfaces are important, metal/organic interfaces, from which charged excitations are injected into the organic material and organic/organic heterostructures, which are used to control current flow by establishing energy barriers in the devices.

In our work on polymer light emitting diodes, we have studied the relative energy levels at the interface between the polymer MEH-PPV and various metals. We discovered that the energy barrier to charged carrier injection varies directly with the work function of the metal. Thus, electrons are easily injected into the polymer from small work function metals and holes are easily injected from large work function metals. This behavior is completely different than in conventional crystalline semiconductors. These observations form the basis for the principles of electronic device design using MEH-PPV and related polymers. They allow, for the first time, a systematic approach to the design of electronic devices based on this class of polymers. This discovery is a first step in the understanding of organic interfaces. We now understand how relative energy levels change as the properties of the organic material are varied so that we can use the methods of organic synthesis to design the organic materials used in the electronic devices. 


\section{Importance to LANL's Science and Technology Base and National R\&D Needs}

Electronic transport in organic and biomolecular materials will be one of the major thrust areas in condensed matter physics in the next century. The basic physical processes in these materials have a number of common themes. The materials are flexible, polarizible, correlated and disordered. They are quite different from the crystalline solids on which most efforts in condensed matter physics have concentrated until now. The phenomenology of these materials is more complex and richer than that of more conventional crystalline materials. New approaches and ideas will be necessary to understand their properties. It is important to understand these kinds of materials because they occur in a wide variety of important physical systems. The work described here is directed toward a particular class of conjugated organic materials, but the principles governing molecular structure-electronic properties relations in these organic materials will have a close analog in biomolecular materials. Therefore, an understanding of these principles will have wide scientific impact.

Electronics based on organic materials is a major emerging technological field. Organic electronic materials can be economically processed over large areas and therefore they have important advantages for large-area electronic applications such as displays and for optical communications. Light emitting diodes, transistors, photodetectors, electro-optic modulators and waveguides are examples of promising device applications of these materials. The basic science of organic electronic materials and of electronic devices fabricated from them is beginning to be understood. An increased understanding of these materials and devices will have an immediate and dramatic technological impact. Light emitting diodes are presently the most successful organic based electronic device. We are currently collaborating with HewlettPackard, UNIAX Inc., the University of Texas at Dallas, and the University of California at Santa Barbara to develop polymer light emitting diodes. These technology development programs were funded by DOE and DARPA. The work described here provides the scientific basis for technology development programs in this area.

\section{Scientific Approach and Accomplishments}

Organic light emitting diodes (LEDs) consist of a thin (typically about 100nm) layer of a conjugated luminescent organic material sandwiched between metallic contacts. When a sufficiently large bias is applied across the contacts, electron polarons are injected from one contact and hole polarons are injected from the other. The electron and hole polarons (electrons and holes for short) recombine in the organic material emitting light. Because organic LEDs 
have potential fabrication, performance and cost advantages, they are being widely investigated for possible display applications. The organic materials used to fabricate organic LEDs are undoped and quite insulating, and current flow in the devices results from carriers that are injected from the contacts. The ideal Schottky model describes the Schottky energy barriers at the interface between metals and conjugated organic materials, and the choice of metals used to form the contacts for organic LEDs is important. For efficient charge injection, high workfunction metals must be used for the hole injecting contact and low workfunction metals must be used for the electron injecting contact.

The mechanism limiting the current flow in an organic LED depends on the device parameters such as the Schottky energy barriers of the contacts, the device length, and the carrier mobilities. It is useful to consider organic diode structures in which one carrier type (i.e., either electrons or holes) dominates the current flow in order to clarify the device operation in a relatively simple situation. Such single carrier structures can be fabricated by choosing the contacts so that the energy barrier for injection of one kind of carrier is much larger than for the other. There have been extensive studies of current flow in the early semiconductor literature. Many of the basic principles of these studies can be expected to apply to the case of organic LEDs, but the range of validity and values of the device parameters for which various limiting cases are appropriate must be established. It has been shown that space-charge-limited current flow can be achieved in organic LEDs. In other organic devices the current flow was clearly injection limited. Because of the disorder present in the amorphous materials that are used to make organic LEDs, the carrier mobilities are strongly field dependent.

We describe a unified device model for single-layer organic LEDs which includes charge injection, transport and space charge effects in the organic material. The model can describe both injection-limited and space-charge-limited cases and the transition between them. We specifically consider cases in which the energy barrier to injection of electrons is much larger than for holes so that holes dominate the current flow in the device. Charge injection into the organic material can occur by thermionic emission and by tunneling. For Schottky energy barriers less than about $0.3-0.4 \mathrm{eV}$, for typical organic LED device parameters, we find that the current flow is space charge limited and the electric field in the structure is highly nonuniform. For larger energy barriers we find that the current flow is injection limited. In this injection-limited regime, the net injected charge density is relatively small, the electric field in the structure is nearly uniform, and space charge effects are not important. At smaller bias in the injected limited regime, thermionic emission is the dominant injection mechanism. For this case the thermionic-emission injection current and a backward flowing interface recombination current combine to establish a quasi-equilibrium carrier density. 
The net device current is determined by the drift of these carriers in the nearly constant electric field. The net device current is much smaller than either the thermionic emission or interface recombination current, which nearly cancel. At higher bias the injection is dominated by tunneling. The bias at which tunneling exceeds thermionic emission depends on the size of the Schottky energy barrier. When tunneling is the dominant injection mechanism, a combination of tunneling injection current and the backflowing interface recombination current combine to establish the carrier density. The net device current is determined by the drift of these carriers in the nearly constant electric field. The net device current is much smaller than either the tunneling or interface recombination current, which nearly cancel. We compare the model results with experimental measurements on devices fabricated using the electroluminescent conjugated polymer poly[2-methoxy, 5-(2'-ethyl-hexyloxy)-1,4-phenylene vinylene] (MEH-PPV), which by changing the contacts can show either injection-limited behavior (ITO contacts) or space-charge-limited behavior (Au contacts).

The device current of organic light emitting diodes is determined by the injection, transport, and recombination of charge carriers. Charge injection depends on the Schottky energy barrier between the contact and the organic material. Charge transport and recombination depend on the material properties of the organic film. We consider structures where the injection processes at the contacts, the transport properties of carriers in the organic film, and the carrier recombination processes can be separated to as large a degree as possible. We present results for current-voltage characteristics of single organic layer LEDs consisting of metal_A/MEH-PPV/metal_B (denoted metal_A/metal_B) structures where MEH-PPV stands for poly[2-methoxy, 5-(2'-ethyl-hexyloxy)-1,4-phenylene vinylene] . We select the contact metals of the devices so that either electrons or holes dominate the current flow. We use the results of these single carrier structures to separately determine the mobility parameters of the two carrier types. We then select the contact metals so that both electrons and holes are injected and use the single-carrier mobility parameters to describe the measured current voltage characteristics of the bipolar devices.

Carrier transport in organic films can be studied in a relatively simple situation by considering single organic layer structures in which the contacts are selected to provide space charge limited current for one carrier type and prevent efficient injection of the other carrier type. The current is then due to the space charge limited current of one carrier and depends only on the bulk transport properties of the organic film for that carrier. Carrier injection can be studied in a relatively simple situation by considering a series of single organic layer structures in which only one contact efficiently injects carriers and the Schottky energy barrier to charge injection of that contact is varied by changing the contacting metal. Carrier recombination affects the injected carrier density and hence the current-voltage (I-V) 
characteristics of bipolar devices. Carrier recombination also determines the luminescence efficiencies of devices, however quantitative evaluation in single layer devices is complicated by cavity effects, dipole quenching near the electrodes, and extrinsic nonradiative recombination centers, and is the topic of future studies.

We first consider $\mathrm{Ca} / \mathrm{Ca}$ electron only structures as a function of polymer thickness. Calcium provides a space charge limited electron contact. We describe the measured I-V characteristics with a device model and extract the electron mobility. For hole injecting structures in MEH-PPV, the device behavior can be varied from space-charge-limited current for small energy barrier contacts such as gold and platinum, to contact-limited current for large energy barrier contacts such as copper or aluminum. We consider $\mathrm{Au} / \mathrm{Al}$ and $\mathrm{Cu} / \mathrm{Al}$ hole only devices. The holes are injected from the gold or copper contacts. We use previously determined Schottky energy barriers for these contacts to MEH-PPV, describe the measured I$\mathrm{V}$ characteristics using a device model, and extract the hole mobility. Finally, we consider $\mathrm{P} / \mathrm{Ca}$ and $\mathrm{Cu} / \mathrm{Ca}$ bipolar devices. Using the mobility parameters determined from the electron only and the hole only structures, the device model successfully describes the measured I-V curves for these bipolar devices with different thicknesses and injection barriers without additional fitting parameters.

The device model considers charge injection from the metal into the organic by thermionic emission and a backflowing interface recombination, which is the time-reversed process of thermionic emission. Charge transport is described by time-dependent continuity equations, with field-dependent carrier mobilities, and a drift-diffusion form for the current coupled to Poisson's equation. Carrier recombination is bimolecular with a Langevin form for the kinetic coefficient. Most of the input parameters required by the model can be measured directly. Capacitance measurements insure that the devices are fully depleted, and that device current is due to injected charge. The device thickness is measured by a Dektak profilometer and the Schottky energy barriers to injection. Time of flight (TOF) measurements on organic materials have found a strongly field-dependent carrier mobility that can be parameterized by the form $\mu=\mu_{0} \exp \sqrt{\mathrm{E} / \mathrm{E}_{0}}$. This form has also been derived theoretically for conduction through a spatially correlated gaussian density of states (DOS). The current-voltage data for the single carrier structures is described by the device model using $\mu_{0}$ and $\mathrm{E}_{0}$ as fitting parameters. The device thickness and energy barriers are constrained to be within the error of their independently measured values. The mobility parameters extracted from the single carrier structures are then used to describe the bipolar devices.

Both electron-phonon and electron-electron interactions play important roles in determining the nature of the fundamental electronic excitations of conjugated polymers. The energies of these excitations are under intense debate. In poly(p-phenylene vinylene) (PPV) 
and its derivatives (e.g. MEH-PPV), the exciton binding energy has been reported to be as high as $1.1 \mathrm{eV}$ or as low as $25 \mathrm{meV}$ and the stability of intrinsic bipolarons is arguable. Interest in these issues has increased with the discovery of polymer light-emitting diodes fabricated from PPV and its derivatives. We describe the results of a series of electroabsorption and internal photoemission measurements on metal/polymer/metal structures which directly determine the single particle (polaron) and charge transfer (bipolaron) energy gaps. Combined with a measurement of the exciton absorption peak, these results determine the exciton and bipolaron binding energies.

We use electroabsorption to measure the built-in electric field in metal/polymer/metal structures. The maximum built-in electric field in the polymer is determined by the charge transfer energy gap; the minimum difference between the chemical potential of negatively and positively charged intrinsic excitations. The maximum built-in field is sensitive to both single particle and multi-particle (bipolaron) states. We find a charge transfer energy gap of at least $2.35 \mathrm{eV}$ for MEH-PPV.

We use internal photoemission to measure electron and hole Schottky barriers at metal/polymer junctions. The electron (hole) Schottky barrier is the energy difference between the Fermi level of the metal and the lowest energy single electron (hole) polaron state of the polymer. The single-particle energy gap is the sum of the electron and hole Schottky barriers, i.e. the sum of the energies required to inject an electron and to inject a hole into the polymer from a fixed Fermi sea. By measuring electron and hole Schottky barriers in the same structure, we find a single-particle energy gap of $2.45 \mathrm{eV}$ for MEH-PPV.

The exciton binding energy is the energy difference between the single particle energy gap and the exciton absorption peak, and the bipolaron binding energy is the difference between the single-particle and charge-transfer energy gaps. For MEH-PPV, with an exciton absorption peak of $2.25 \mathrm{eV}$, our measurements yield an exciton binding energy of $0.2 \mathrm{eV}$ and a bipolaron binding energy of less than $0.1 \mathrm{eV}$.

Chemical reactions between the metal and the polymer could lead to interface states that pin the Schottky barrier as occurs in many inorganic semiconductors. Interface pinning would not influence the interpretation of the internal photoemission measurement of the single-particle gap, because it just redistributes the electron and hole barriers with their sum remaining unchanged. Interface pinning could reduce the maximum built-in field in the polymer, but not increase it. We find that the maximum built-in field is nearly equal to the single-particle gap so that the charge-transfer gap is close to the single-particle gap. The systematics of our measurements (i. e., the observed Schottky barriers directly track the metal work function within the single particle gap) show that the metals investigated do not introduce interface states in the single-particle gap that pin the Schottky barrier. This does not imply that the metals and 
the polymer do not interact, there is considerable evidence that they do react chemically, but only that these interactions do not pin the Schottky barrier.

The metal/polymer/metal structures consisted of a thin, semi-transparent metal on a glass substrate, an undoped polymer layer $\sim 50 \mathrm{~nm}$ thick, and a top thick metal contact. The structures were fabricated in an inert atmosphere using spin casting to produce the polymer film. The top metal contact was deposited through a shadow mask to produce diodes with active areas of $4 \cdot 10^{-2} \mathrm{~cm}^{2}$. The structures were loaded into a vacuum cryostat and measured at room temperature. The quantum efficiencies of light-emitting diodes fabricated by these procedures are in good agreement with the best published values.

We described a unified device model for single-layer organic LEDs which includes charge injection, transport and space charge effects in the organic material. We specifically considered cases in which the energy barrier to injection of electrons is much larger than for holes so that holes dominate the current flow in the device. Charge injection into the organic material can occur by thermionic emission and by tunneling. For Schottky energy barriers less than about $0.3-0.4 \mathrm{eV}$, for typical organic LED device parameters, we found that the current flow is space charge limited and the electric field in the structure is highly nonuniform. The calculations here include carrier diffusion and field-dependent mobilities and therefore they do not reduce to the simple analytic form often used to describe the space charge limited regime, which is derived for field-independent mobilities neglecting diffusion. For larger energy barriers we found that the current flow is injection limited. In the injection-limited regime the net injected charge is relatively small, the electric field and carrier density are nearly uniform and space charge effects are not important. Because the carrier density is nearly constant in this regime the device current density is well approximated by the drift component of the current. Experimentally, plotting the current for devices with different lengths as a function of the spatially averaged applied electric field is a test to determine if the structures are injection or space charge limited. In the injection-limited regime, the current for different length devices will nearly coincide when plotted as a function of the averaged applied electric field if the effect of the built-in potential is corrected for or is not too large. In the space charge limited regime, the currents for different length devices will not coincide when plotted in this way.

In the model described here injection currents appear as boundary conditions for carrier continuity equations with a drift-diffusion form for the current densities. In the space charge limited regime, these boundary conditions reduce to the requirement that the carrier densities at the injecting contacts have their equilibrium values. In this case, the equilibrium carrier density at an injecting contact is very large and the current flow in the structure does not significantly perturb such a large carrier density. In the injection limited regime when the bias is small enough that injection by thermionic emission is larger than by tunneling, the boundary 
conditions reduce to the requirement that the carrier densities at the injecting contacts are given by quasi-equilibrium values that include image force lowering of the injection barrier.

Thermionic emission and its time-reversed process, interface recombination, set up a quasiequilibrium situation in the contact region that is not significantly disturbed by the current flow in the structure. In both of these limiting cases, all of the complexity of the actual injection processes are eliminated from the device description. Only when tunneling injection becomes significant, so that the organic material in the contact region is not in quasi-equilibrium with the metal contact, does the detailed description of the injection processes become important.

\section{Publications}

1. I. H. Campbell, T. W. Hagler, D. L. Smith and J. P. Ferraris,"Direct Measurement of Conjugated Polymer Electronic Excitation Energies Using Metal/Polymer/Metal Structures", Phys. Rev. Lett. 76, 1900 (1996).

2. P. S. Davids, A. Saxena and D. L. Smith, "Bipolaron Lattice Formation at Metal-Polymer Interfaces", Phys. Rev. B53, 4823 (1996).

3. I. H. Campbell, J. P. Ferraris, T. W. Hagler, M. D. Joswick, D. L. Smith and I. D. Parker, "Measuring Internal Electric Fields in Organic Light-Emitting Diodes Using Electroabsorption Spectroscopy", Polymers for Advanced Technologies $\underline{8}, 417$ (1997).

4. C. M. Heller, I. H. Campbell, B. K. Laurich, D. L. Smith, D. D. C. Bradley, P. L. Burn, J. P. Ferraris, and K. Mullen, "Solid State Concentration-Effects on the Optical Absorption and Emission of Poly (p-phenylene vinylene) Related Materials", Phys. Rev. B54, 5516 (1996).

5. I. H. Campbell, P. S. Davids, J. P. Ferraris, T. W. Hagler, C. M. Heller, D. L. Smith and A. Saxena, "Probing Electronic State Charging in Organic Electronic Devices Using Electroabsorption Spectroscopy", Synth. Metals 무, 105 (1996).

6. P. S. Davids, Sh. M. Kogan, D. L. Smith and I. D. Parker, "Charge Injection in Organic Light-Emitting Diodes: Tunneling into Low Mobility Materials", Appl. Phys. Lett. 69, 2270 (1996).

7. D. L. Smith and Sh. M. Kogan, "Theory of Ballistic Electron Emission Microscopy of Buried Semiconductor Heterojunctions", Phys. Rev B54, 10354 (1996).

8. C. M. Heller, I. H. Campbell, N. N. Barashkov, and J. P. Ferraris, "Chemical Potential Pinning Due to Equilibruim Electron Transfer at Metal/C60- Doped Polymer Interfaces", J. Appl. Phys. 1ㅗ, 3227 (1997).

9. E. Y. Lee, V. Narayanamurti and D. L. Smith, "Monte Carlo Simulation of BEEM Imaging and Spectroscopy of Buried Mesoscopic Structures", Phys. Rev. B55, 16033 (1997).

10. Z. G. Yu, D. L. Smith, A. Saxena, and A. R. Bishop, "Effects of Lattice Fluctuations on Electronic Transmission in Metal/Conjugated-Oligomer/Metal Structures", Phys. Rev. B56, 6494 (1997). 
11. D. L. Smith, E. Y. Lee and V. Narayanamurti, "Theory of Ballistic Electron Emission Microscopy for Nonepitaxial Metal/Semiconductor Interfaces", Phys. Rev. Lett. 80, 2433 1998).

12. P. S. Davids, I. H. Campbell, and D. L. Smith, "Device Model for Single Carrier Organic Diodes", J. Appl. Phys. $\underline{82}, 6319$ (1997).

13. J. Cornil, D. Beljonne, C. M. Heller, I. H. Campbell, B. K. Laurich, D. L. Smith, D. D. C. Bradley, K. Mullen, and J. L. Bredas, "Photoluminescence Spectra of Oligo-

Paraphenylenevinylenes: A Joint Theoretical and Experimental Characterization", Chem. Phys. Lett., 278, 139 (1997).

14. Z. G. Yu, D. L. Smith, A. Saxena, and A. R. Bishop, "Electronic Transmission in Conjugated-Oligomer Tunnel Structures: Effects of Lattice Flucations", J. Phys.: Cond. Mat. $10,617(1998)$.

15. I. H. Campbell, P. S. Davids, D. L. Smith, N. N. Barashkov, and J. P. Ferraris, "The Schottky Energy Barrier Dependence of Organic Light-Emitting Diodes", Appl. Phys. Lett. 72, 1863 (1998).

16. A. Saxena, D. L. Smith, Z. G. Yu and A. R. Bishop, "Electronic Transmission Across A Metal/Conjugated-Oligomer/Metal Structure: Role Of Intrinsic Disorder", Opt. Mat. 9, 461 (1998).

17. B. K. Crone, P. S. Davids, I. H. Campbell and D. L. Smith, "Device Model Investigation of Single Layer Organic Light Emitting Diodes" J. Appl. Phys. 4, 833 (1998).

18. Z. Y. Yu, D. L. Smith A. Saxena and A. R. Bishop, "Dynamics of Electronic Transport in Metal/Organic/Metal Structures", J. Phys.: Cond. Mat. (accepted).

19. B. K. Crone, I. H. Campbell, P. S. Davids, D. L. Smith,. C. J. Neef and J. P. Ferraris, "Charge Injection and Transport in Single Layer Light Emitting Diodes", Appl. Phys Lett. 73, 3162 (1998).

20. I. H. Campbell and D. L. Smith, "Schottky Energy Barriers and Charge Injection in Alq/Metal Structures", Appl. Phys. Lett. (accepted).

21. I. H. Campbell and D. L. Smith, "Physics of Polymer Light Emitting Diodes", in Semiconducting Polymers-Chemistry, Physics and Engineering, edited by Hadziioannuo and Van Hutten, (VHC-Wiley) (accepted). 